Journal of

Synchrotron

Radiation

ISSN 1600-5775

\title{
Study on the reflectivity properties of spherically bent analyser crystals
}

\author{
Ari-Pekka Honkanen, Roberto Verbeni, Laura Simonelli, Marco Moretti \\ Sala, Giulio Monaco and Simo Huotari
}

J. Synchrotron Rad. (2014). 21, 104-110

Copyright (C) International Union of Crystallography

Author(s) of this paper may load this reprint on their own web site or institutional repository provided that this cover page is retained. Republication of this article or its storage in electronic databases other than as specified above is not permitted without prior permission in writing from the IUCr.

For further information see http://journals.iucr.org/services/authorrights.html

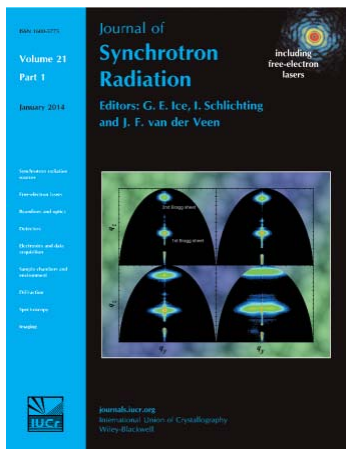

Synchrotron radiation research is rapidly expanding with many new sources of radiation being created globally. Synchrotron radiation plays a leading role in pure science and in emerging technologies. The Journal of Synchrotron Radiation provides comprehensive coverage of the entire field of synchrotron radiation research including instrumentation, theory, computing and scientific applications in areas such as biology, nanoscience and materials science. Rapid publication ensures an up-to-date information resource for scientists and engineers in the field.

Crystallography Journals Online is available from journals.iucr.org 
Journal of

Synchrotron

Radiation

ISSN 1600-5775

Received 13 March 2013

Accepted 9 August 2013

\section{Study on the reflectivity properties of spherically bent analyser crystals}

\author{
Ari-Pekka Honkanen, ${ }^{a *}$ Roberto Verbeni, ${ }^{\mathbf{b}}$ Laura Simonelli, ${ }^{\mathbf{b}}$ Marco Moretti Sala, \\ Giulio Monaco $^{\mathrm{b}, \mathrm{c}}$ and Simo Huotari ${ }^{\mathrm{a}}$
}

${ }^{\mathbf{a}}$ Department of Physics, PO Box 64, Fl-00014 Helsinki, Finland, ${ }^{\mathbf{b}}$ European Synchrotron Radiation Facility, BP 220, F-38043 Grenoble Cedex, France, and ${ }^{\mathbf{c}}$ Physics Department, University of Trento, Via Sommarive 14, 38123 Povo (TN), Italy. *E-mail: ari-pekka.honkanen@helsinki.fi

\begin{abstract}
Theoretical and experimental studies are presented on properties of spherically bent analyser crystals for high-resolution X-ray spectrometry. A correction to the bent-crystal strain field owing to its finite surface area is derived. The results are used to explain the reflectivity curves and anisotropic properties of $\mathrm{Si}(660)$ and $\mathrm{Si}(553)$ analysers in near-backscattering geometry. The results from the calculation agree very well with experimental results obtained using an inelastic $\mathrm{X}$-ray scattering synchrotron beamline.
\end{abstract}

(C) 2014 International Union of Crystallography

\section{Introduction}

$\mathrm{X}$-ray spectroscopy is a highly versatile tool in physics, chemistry and materials science. Being non-destructive, and selective to element, chemical bond, spin state and even atomic site makes high-resolution X-ray spectroscopy a unique probe for structural and chemical analysis. Studies may range from core-level spectroscopy (de Groot \& Kotani, 2008) to valence-electron charge, spin and orbital dynamics in resonant and non-resonant inelastic X-ray scattering (IXS) (Schülke, 2007).

Highest energy resolution today in the hard X-ray regime $(5-20 \mathrm{keV})$ is achieved with wavelength-dispersive spectrometers that are based on diffractive crystal optics (Shvyd'ko, 2004). The heart of the instrument is an analyser crystal, which has to collect radiation scattered or emitted by the sample to a relatively large solid angle, and reflect the wavelengthanalysed radiation onto a detector. An efficient analyser crystal has to accept radiation with a very large divergence (e.g. $100 \mathrm{mrad}$, possibly up to $200 \mathrm{mrad}$ ). To achieve this goal, different curved focusing or dispersive designs have been used for almost a century (DuMond \& Kirkpatrick, 1930; Johann, 1931; Johansson, 1932, 1935; Cauchois, 1932; von Hamos, 1932, 1933). All of the designs are being used very actively today for high-resolution X-ray spectroscopy at synchrotrons and at X-ray free-electron lasers (Alonso-Mori et al., 2012; Sakurai et al., 2011; Shvyd'ko et al., 2012). Single-wavelength selection with a relatively high resolving power has been achieved by using designs based on the Rowland circle using the Johann geometry. When Bragg angles can be chosen to be between 70 and $90^{\circ}$, relatively good focusing can be achieved using spherical analyser crystals in the non-dispersive direction as well. For X-ray emission spectroscopy and IXS spectroscopy, spherically bent crystals are indeed widely used
(Verbeni et al., 2009; Journel et al., 2009; Fister et al., 2006; Hazemann et al., 2009; Welter et al., 2005; Kleymenov et al., 2011; Llorens et al., 2012; Collart et al., 2005; Shvyd'ko et al., 2012).

Bending a diffractive crystal results in elastic deformations that affect its reflectivity curve (Penning \& Polder, 1961; Takagi, 1962, 1969; Taupin, 1964; Kuriyama, 1967). Such strains can be avoided in designs that in one way or another approximate a spherical shape such as in so-called diced (also called pixelated) analyser crystals (Masciovecchio et al., 1996; Said et al., 2011) or in recent miniature X-ray emission spectrometers (Pacold et al., 2012; Mattern et al., 2012). Even the contribution to the energy resolution due to the geometrical deviation from a perfect spherical shape of a diced analyser crystal can be corrected for (Huotari et al., 2005, 2006). Diced crystals do offer at the moment the highest energy resolution $(E / \Delta E)>2 \times 10^{7}$ for Johann-type hard X-ray spectrometers. However, in some cases elastic bending of the analyser crystal can be advantageous. An increased bandwidth may increase efficiency, which can be used in cases when higher intensity is more desirable than very high resolving power. A bent crystal also offers point-to-point focusing, which can be used to achieve three-dimensional imaging of a heterogeneous sample (Huotari et al., 2011).

In order to understand the resulting bent-crystal reflectivity curves, the diffraction properties can be calculated by solving the Takagi-Taupin equations when the strain field is known (Sanchez del Rio et al., 1997) or by using so-called lamellar models (Erola et al., 1990; del Rio et al., 2004). This has been achieved in the case of simplified approximations for the strain field, e.g. spherical or cylindrical bending free from angular compression. For real macroscopic crystals with anisotropic elastic properties, the full solution of the real strain field induced by bending requires a finite-element-method 
approach. However, that task, especially combined with solutions to Takagi-Taupin equations, is computationally expensive. This is unfortunate, since it is well known that experimentally measured bandwidths for macroscopic analysers are generally inferior to those predicted for simplified spherical bending, and even generally depend on the active crystal aperture size (Verbeni et al., 2005, 2009). Different paths to achieve results closer to those expected from simplified spherical bending have been taken such as increasing bending radii (Collart et al., 2005) and introducing stress-relief cuts (Verbeni et al., 2009; Qian, 2013). However, understanding the reflectivity curve and its behaviour on analyser crystal size and bending radius would be indispensable in order to construct fully optimized spectrometers with the highest performance.

In this article we present an attractive analytical approach to estimate the strain field for thin but large-area spherical analyser crystals. We use the obtained strain field to estimate the bandwidth of such finite-sized crystals. We obtain excellent quantitative agreement with experimental results of $\mathrm{Si}(660)$ and $\operatorname{Si}(553)$ analyser crystals with thicknesses of $<300 \mu \mathrm{m}$, diameters of $100 \mathrm{~mm}$ and bending radii of $1 \mathrm{~m}$.

\section{Theory}

Exact calculation of the deformation field of a bent crystal should be performed using finite-element methods. However, assuming the wafer to be thin, we can make numerical predictions with relatively little effort for arbitrary crystal materials and orientations. It turns out that this approximation is actually surprisingly accurate in predicting diffraction properties of real spherically bent analyser crystals.

\subsection{Theory of elasticity}

Suppose that the position of the arbitrary point in an undeformed body is given by vector $\mathbf{x}$ and after deformation by $\mathbf{x}^{\prime}$. The displacement vector $\mathbf{u}$ is defined to be

$$
\mathbf{u}=\mathbf{x}^{\prime}-\mathbf{x} .
$$

Within the theory of linear elasticity (Landau \& Lifshitz, 1986), the deformations are small and the strain tensor $\varepsilon_{i j}$ is given by

$$
\varepsilon_{i j}=\frac{1}{2}\left(\frac{\partial u_{i}}{\partial x_{j}}+\frac{\partial u_{j}}{\partial x_{i}}\right)
$$

where the indices $i, j \in x, y, z$ refer to the $x$-, $y$ - and $z$-directions in the Cartesian coordinate system, respectively.

The stresses and the strains of a body in general are connected by Hooke's law as follows,

$$
\sigma_{i j}=\sum_{k, l} c_{i j k l} \varepsilon_{k l} \quad \text { and } \quad \varepsilon_{i j}=\sum_{k, l} s_{i j k l} \sigma_{k l},
$$

where $\sigma$ and $\varepsilon$ are the stress and the strain tensors and $c$ and $s$ are the stiffness and the compliance tensors, respectively. For elastically isotropic material the former are reduced to (Landau \& Lifshitz, 1986)

$$
\sigma_{i j}=\frac{Y}{1+v}\left(\varepsilon_{i j}+\frac{v}{1-2 v} \delta_{i j} \sum_{k=1}^{3} \varepsilon_{k k}\right)
$$

and

$$
\varepsilon_{i j}=\frac{1+v}{Y} \sigma_{i j}-\frac{v}{Y} \delta_{i j} \sum_{k=1}^{3} \sigma_{k k},
$$

where $Y$ is Young's modulus, $v$ is Poisson's ratio and $\delta_{i j}$ is Kronecker's symbol.

Owing to the symmetries of the aforementioned tensors, Hooke's law in (3) can also be written in the matrix form

$$
\boldsymbol{\sigma}=C \boldsymbol{\varepsilon} \quad \text { and } \quad \boldsymbol{\varepsilon}=S \boldsymbol{\sigma},
$$

where $\boldsymbol{\sigma}$ and $\boldsymbol{\varepsilon}$ are six-element column vectors corresponding to $\sigma$ and $\varepsilon$. $C$ and $S$ are $6 \times 6$-matrices corresponding to $c$ and $s$. Since the matrix representation is not unique, we have chosen to use the Voigt representation (see, for example, Fast et al., 1995) in this paper.

In the equilibrium, the stress tensor $\sigma$ must fulfil (in cylindrical coordinates) (Nowacki, 1963)

$$
\begin{gathered}
\frac{\partial \sigma_{r r}}{\partial r}+\frac{1}{r} \frac{\partial \sigma_{r \varphi}}{\partial \varphi}+\frac{\partial \sigma_{r z}}{\partial z}+\frac{1}{r}\left(\sigma_{r r}-\sigma_{\varphi \varphi}\right)=0, \\
\frac{\partial \sigma_{r \varphi}}{\partial r}+\frac{1}{r} \frac{\partial \sigma_{\varphi \varphi}}{\partial \varphi}+\frac{\partial \sigma_{\varphi z}}{\partial z}+\frac{2}{r} \sigma_{r \varphi}=0, \\
\frac{\partial \sigma_{r z}}{\partial r}+\frac{1}{r} \frac{\partial \sigma_{\varphi z}}{\partial \varphi}+\frac{\partial \sigma_{z z}}{\partial z}+\frac{1}{r} \sigma_{r z}=0 .
\end{gathered}
$$

According to Newton's third law, external forces acting on a body and the stress tensor at boundaries of a body obey the relation

$$
P_{i}=\sum_{k} \sigma_{i k} n_{k},
$$

where $P_{i}$ is the $i$-component of the force per unit surface area and $n_{k}$ is the $k$-component of the surface normal vector.

\subsection{Thin wafer approximation}

In order to obtain an important intermediate result, we assume for now that the crystal wafer is elastically isotropic. In this subsection we show that in scope of this approximation, $\varepsilon_{z z}$, which is used in reflectivity calculations, can be separated into two terms, the first one being solely the function of $z$ and the other being the function of the $x$ and $y$. The isotropic approximation will be relaxed later in order to calculate the $x$ and $y$-dependence of $\varepsilon_{z z}$. It will be also shown that in the range of the cases studied here, this approximation gives good agreement with experiment.

Let us assume a round crystal wafer with radius $L$ and thickness $d$ and a Cartesian coordinate $\operatorname{system}(x, y, z)$ which is chosen so that its origin lies in the center of the wafer and $z$ direction is normal to the surface of the wafer. We further assume that the wafer is thin, i.e. $d \ll L$, and therefore the forces needed to bend it are negligible compared with the internal stresses. Thus the $P_{i}$ in (10) are approximately zero. Since the deformation of the wafer is small, we can assume 
that the normal vectors of the deformed upper and the bottom surface are approximately in the $z$-direction. According to $(10)$,

$$
\sigma_{x z}=\sigma_{y z}=\sigma_{z z}=0 .
$$

Since the wafer is thin, we can assume that this holds, at least approximately, inside the wafer as well. By substitution of (11) into (5), we find that

$$
\varepsilon_{x z}=\varepsilon_{z x}=0 \quad \text { and } \quad \varepsilon_{y z}=\varepsilon_{z y}=0 .
$$

Now, using (2) and (12), the horizontal components of the displacement vector are found to be

$$
\begin{aligned}
& u_{x}=-\int \frac{\partial u_{z}}{\partial x} \mathrm{~d} z+A(x, y), \\
& u_{y}=-\int \frac{\partial u_{z}}{\partial y} \mathrm{~d} z+B(x, y),
\end{aligned}
$$

where $A(x, y)$ and $B(x, y)$ are constants of integration with respect to $z$.

The vertical displacement of the bottom surface of the analyser $u_{z}(x, y,-d / 2)$ due to spherical bending is approximately given by

$$
u_{z}(x, y,-d / 2)=\frac{x^{2}+y^{2}}{2 R},
$$

where $R$ is the radius of the sphere for $\left(x^{2}+y^{2}\right)^{1 / 2} \leq L \ll R$. Since the wafer is thin we can assume that the partial derivatives $\partial u_{z} / \partial z$ and $\partial u_{z} / \partial y$ in (13) and (14) do not depend strongly on $z$. Thus they can be approximated with respective partial derivatives of (15). Combining (13), (14) and (15), we obtain

$$
\begin{aligned}
& u_{x}=-\frac{x z}{R}+A(x, y), \\
& u_{y}=-\frac{y z}{R}+B(x, y) .
\end{aligned}
$$

Substituting the former into (2), we find the normal strains in the $x$ - and $y$-direction to be

$$
\begin{aligned}
& \varepsilon_{x x}=-\frac{z}{R}+\frac{\partial A(x, y)}{\partial x}, \\
& \varepsilon_{y y}=-\frac{z}{R}+\frac{\partial B(x, y)}{\partial y} .
\end{aligned}
$$

Since $\sigma_{z z}=0$, from (4) it follows that

$$
(1-v) \varepsilon_{z z}+v\left(\varepsilon_{x x}+\varepsilon_{y y}\right)=0 .
$$

Substituting (18) and (19) into (20), we find that the total strain in the $z$-direction $\varepsilon_{z z}$ can be separated into a function of vertical coordinate $z$ and a function of horizontal coordinates $x$ and $y$, so that

$$
\varepsilon_{z z}=\varepsilon_{z z}^{\mathrm{v}}(z)+\varepsilon_{z z}^{\mathrm{h}}(x, y),
$$

where

$$
\varepsilon_{z z}^{\mathrm{v}}(z)=\frac{2 v}{1-v} \frac{z}{R}
$$

and $\varepsilon_{z z}^{\mathrm{h}}(x, y)$ is a yet-to-be-solved function of $A(x, y)$ and $B(x, y)$. For small deformations, $A(x, y)$ and $B(x, y)$ can be usually set to zero, since they are of second-order in terms of $u_{z}$, whereas integrals in (13) and (14) are of first-order (Landau \& Lifshitz, 1986). However, experiments have shown that the shape of the reflectivity curve is different for various sized active surface areas (Verbeni et al., 2005, 2009). This implies that the strain field cannot be assumed to be independent of $x$ and $y$. An approximate expression for the $x$ - and $y$-dependency is derived in the following subsection.

\subsection{Infinitely thin wafer}

Let us examine the limit of an infinitely thin wafer $(h \rightarrow 0)$. Now, in (21), $\varepsilon_{z z} \rightarrow \varepsilon_{z z}^{\mathrm{h}}(x, y)$. For solving $\varepsilon_{z z}^{\mathrm{h}}$, we will apply the more general case of anisotropic equations of elasticity.

Prior to the spherical bending, a circle (that is concentric with the wafer) with a radius $l \leq L$ has a circumference of $2 \pi l$. During the bending process, the circumference has to contract down to $2 \pi l^{\prime}$ in order to make the flat wafer fit on the spherical surface, leading to an angular compression. This is illustrated in Fig. 1. The mean strain $\left\langle\varepsilon_{\varphi \varphi}\right\rangle$ in the angular direction is then

$$
\left\langle\varepsilon_{\varphi \varphi}\right\rangle=\frac{2 \pi l^{\prime}}{2 \pi l}-1=\frac{\sin \alpha}{\alpha}-1 \simeq-\frac{\alpha^{2}}{6},
$$

where $\alpha=\arcsin \left(l^{\prime} / R\right)=l / R$. Since $l, l^{\prime} \ll R$, we can write $\alpha \simeq r / R$.

We see from (23) that $\left\langle\varepsilon_{\varphi \varphi}\right\rangle$ is proportional to $r^{2}$. According to Hooke's law, the stress is a linear function of strain. Thus we can make an assumption that the normal stress in the angular direction is

$$
\sigma_{\varphi \varphi}=-D r^{2}
$$

where $D$ is a (positive) proportional factor that depends on the material and the cutting direction of the wafer. Despite the anisotropy of the crystal, $\sigma_{\varphi \varphi}$ is assumed to be independent of $\varphi$, otherwise the crystal would not be in equilibrium and the stress would even itself out. From (10) it follows

$$
\sigma_{r z}=\sigma_{\varphi z}=\sigma_{z z}=0
$$

It is also safe to assume that

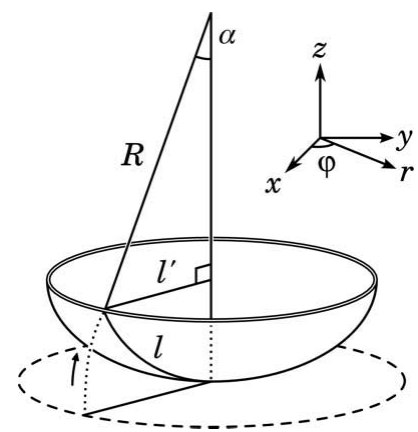

Figure 1

The circumference $2 \pi l$ of a circle with radius $l$ on the undeformed wafer has to contract down to $2 \pi l^{\prime}$ in order to fit on a spherical surface with bending radius $R$. The relation of the used cylindrical coordinate system $(r, \varphi, z)$ to the Cartesian system $(x, y, z)$ is shown on the right. For convenience, the same label for the $z$-direction is used in both systems. 


$$
\sigma_{r \varphi}=0,
$$

since the opposite would correspond to the case in which the wafer is twisted along the $\varphi$-direction. Finally, since the stress is also assumed to be cylindrically symmetric, all the derivatives with respect to $\varphi$ are zero. Thus (7), (8) and (9) reduce to

$$
\frac{\partial \sigma_{r r}}{\partial r}+\frac{1}{r}\left(\sigma_{r r}-\sigma_{\varphi \varphi}\right)=0
$$

Substituting (24) into (27) and solving the differential equation, we find the radial normal stress to be

$$
\sigma_{r r}=\frac{1}{3} \sigma_{\varphi \varphi}=-\frac{D}{3} r^{2} .
$$

In order to solve the strain $\varepsilon_{z z}^{\mathrm{h}}$ in the reflective direction, we make use of the Cartesian coordinate system as depicted in Fig. 2. We denote the local Cartesian axes with $x^{\prime}$ and $y^{\prime}$, thus emphasizing the difference between the local system and the one used in the previous section. The $z^{\prime}$-axis will be referred to simply as $z$, since it is unchanged in the transformation.

From (6) we see that $\varepsilon_{z z}^{\mathrm{h}}$ in Cartesian coordinates is given by $\varepsilon_{z z}^{\mathrm{h}}=S_{31}^{\prime} \sigma_{x^{\prime} x^{\prime}}+S_{32}^{\prime} \sigma_{y^{\prime} y^{\prime}}+S_{33}^{\prime} \sigma_{z z}+S_{34}^{\prime} \sigma_{y^{\prime} z}+S_{35}^{\prime} \sigma_{x^{\prime} z}+S_{36}^{\prime} \sigma_{x^{\prime} y^{\prime}}$,

where $S^{\prime}$ is the rotated compliance matrix. Equations (25) and (26) imply that also in local Cartesian coordinates

$$
\sigma_{x^{\prime} z}=\sigma_{y^{\prime} z}=\sigma_{x^{\prime} y^{\prime}}=\sigma_{z z}=0 .
$$

Substituting the former into (29) we obtain

$$
\varepsilon_{z z}^{\mathrm{h}}=S_{31}^{\prime} \sigma_{x^{\prime} x^{\prime}}+S_{32}^{\prime} \sigma_{y^{\prime} y^{\prime}} .
$$

Since $\sigma_{x^{\prime} x^{\prime}}=\sigma_{r r}$ and $\sigma_{y^{\prime} y^{\prime}}=\sigma_{\varphi \varphi}$, we obtain by substituting (24) and (28) into (31)

$$
\varepsilon_{z z}^{\mathrm{h}}=-D\left(\frac{S_{31}^{\prime}}{3}+S_{32}^{\prime}\right) r^{2} .
$$

To solve coefficient $D$, we must take a look at angular strain $\varepsilon_{\varphi \varphi}$. Making use of the local Cartesian coordinate system, this is given by

$$
\begin{aligned}
\varepsilon_{\varphi \varphi} & =S_{21}^{\prime} \sigma_{x^{\prime} x^{\prime}}+S_{22}^{\prime} \sigma_{y^{\prime} y^{\prime}}+S_{23}^{\prime} \sigma_{z z}+S_{24}^{\prime} \sigma_{y^{\prime} z}+S_{25}^{\prime} \sigma_{x^{\prime} z}+S_{26}^{\prime} \sigma_{x^{\prime} y^{\prime}} \\
& =S_{21}^{\prime} \sigma_{x^{\prime} x^{\prime}}+S_{22}^{\prime} \sigma_{y^{\prime} y^{\prime}}=-D\left(\frac{S_{21}^{\prime}}{3}+S_{22}^{\prime}\right) r^{2}
\end{aligned}
$$

thus the mean value of $\varepsilon_{\varphi \varphi}$ is

\section{Figure 2}

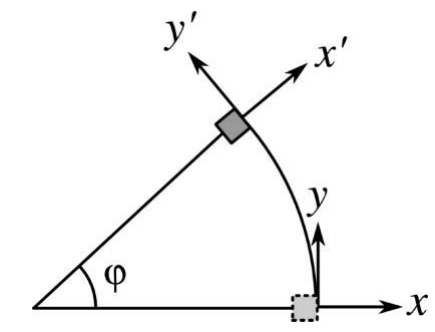

The local Cartesian coordinate system used in calculation of $\varepsilon_{z z}^{\mathrm{h}}$. The coordinate system is rotated about the $z$-axis by an angle $\varphi$, keeping the $x^{\prime}$-axis parallel with the radial component $r$ of the original cylindrical coordinates. The grey square represents a cube of infinitesimal size for which the rotated compliance matrix $S^{\prime}$ is computed.

$$
\left\langle\varepsilon_{\varphi \varphi}\right\rangle=-D\left(\frac{1}{3}\left\langle S_{21}^{\prime}\right\rangle+\left\langle S_{22}^{\prime}\right\rangle\right) r^{2} .
$$

Now, combining (23) and (34) we find that the value of $D$ is

$$
D=\frac{1}{6 R^{2}}\left(\frac{1}{3}\left\langle S_{21}^{\prime}\right\rangle+\left\langle S_{22}^{\prime}\right\rangle\right)^{-1} .
$$

Therefore, the expression for $\varepsilon_{z z}^{\mathrm{h}}$ is found to be

$$
\varepsilon_{z z}^{\mathrm{h}}(\alpha, \varphi)=-\frac{\alpha^{2}}{6} \frac{S_{31}^{\prime}+3 S_{32}^{\prime}}{\left\langle S_{21}^{\prime}\right\rangle+3\left\langle S_{22}^{\prime}\right\rangle} .
$$

For arbitrary rotation matrix $Q$ the components of the rotated compliance tensor $s_{i j k l}^{\prime}$ are given by

$$
s_{i j k l}^{\prime}=\sum_{p, q, r, s} Q_{i p} Q_{j q} Q_{k r} Q_{l s} s_{p q r s},
$$

from which rotated compliance matrix $S^{\prime}$ is then formed according to the Voigt notation. For rotation amount of $\varphi$ around the $z$-axis the rotation matrix $Q_{z}(\varphi)$ is given by

$$
Q_{z}(\varphi)=\left(\begin{array}{ccc}
\cos \varphi & \sin \varphi & 0 \\
-\sin \varphi & \cos \varphi & 0 \\
0 & 0 & 1
\end{array}\right)
$$

$Q_{z}$ rotates the tensor clockwise, which is equivalent to counterclockwise rotation of the primed coordinate system as depicted in Fig. 2. The expressions for terms involving components of $S^{\prime}$ in (36) are thus

$$
\begin{aligned}
S_{31}^{\prime}+3 S_{32}^{\prime}= & 2\left(S_{31}+S_{32}\right) \\
& +\left[\left(S_{32}-S_{31}\right)^{2}+S_{36}^{2}\right]^{1 / 2} \cos (2 \varphi+\beta),
\end{aligned}
$$

where

$$
\beta=\arctan \left(\frac{S_{36}}{S_{32}-S_{31}}\right),
$$

and

$$
\left\langle S_{21}^{\prime}\right\rangle+3\left\langle S_{22}^{\prime}\right\rangle=\frac{5\left(S_{11}+S_{22}\right)+6 S_{21}+S_{66}}{4} .
$$

\subsection{Reflectivity curves}

The calculated strain field can be used to predict the reflectivity curves of any kind of spherical analyser for which the approximations apply. The vertical part of the strain $\varepsilon_{z z}^{\mathrm{v}}$ in (22) is used to calculate the reflectivity curve of the spherically bent crystal without angular compression by solving the depth-dependent Takagi-Taupin equation as described by Gronkowski (1991). The used Poisson's ratios were obtained by averaging $v_{13}^{\prime}=-S_{13}^{\prime} / S_{11}^{\prime}$ over full angle. Since $\varepsilon_{z z}^{\mathrm{h}}$ in (36) is a rather small relative correction (of order $10^{-4}$ ) to the separation of Bragg planes and since it does not vary as a function of $z$, we assume that its effect on the shape of the solution to Takagi-Taupin equations can be neglected. However, we do assume that the small separation of Bragg planes affects the energy of the reflected photons so that the Takagi-Taupin curve at $(r, \varphi)$ is shifted along the energy axis by an amount 

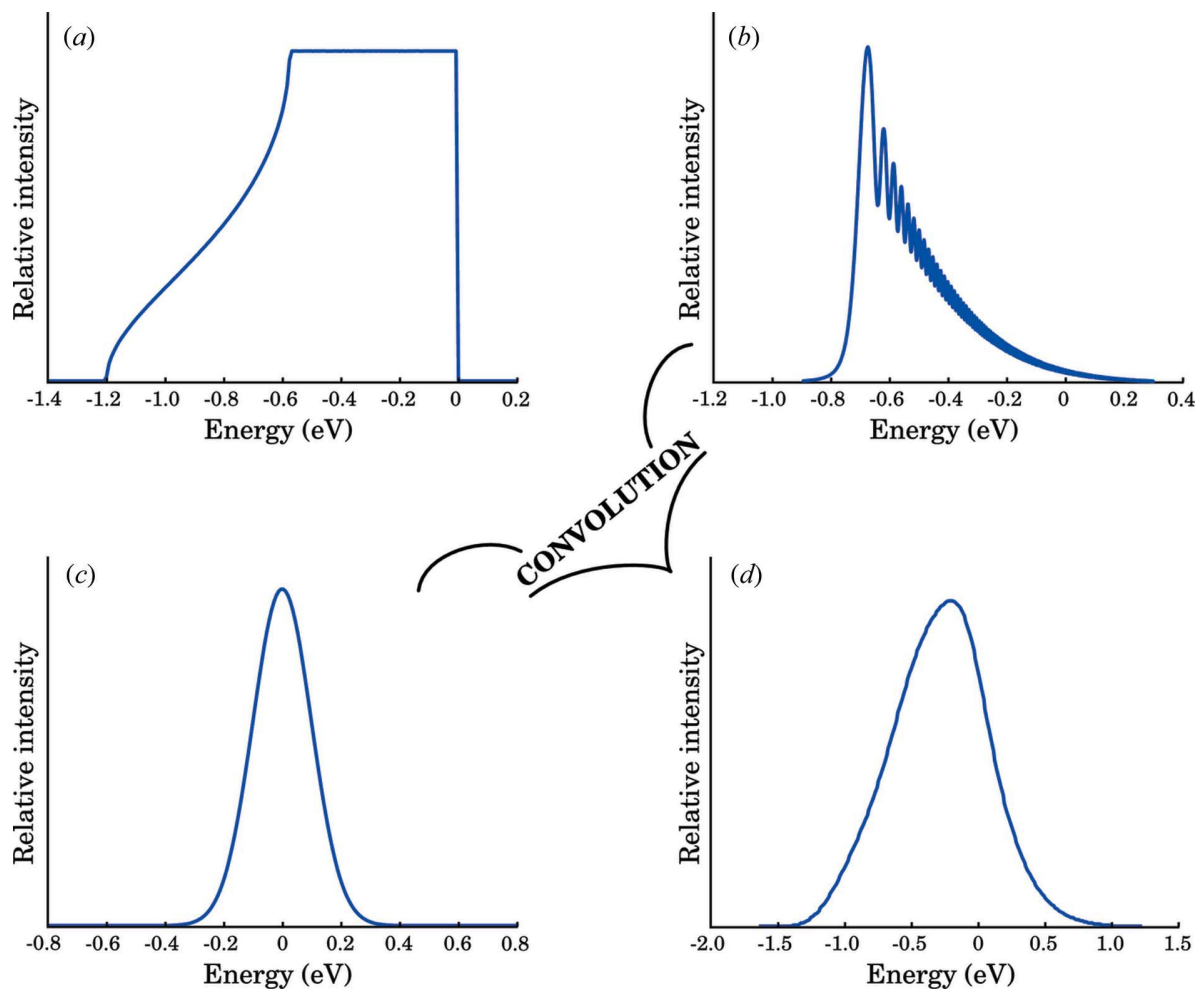

Figure 3

Composition of the theoretical reflectivity curve for a $\mathrm{Si}(660)$ analyser with $86 \mathrm{~mm}$ diameter. (a) Distribution of the shift of the reflected photon energy given by equation (42). (b) Reflectivity for a spherically bent crystal without angular compression computed from Takagi-Taupin equations. $(c)$ Simulated bandwidth curve of the incoming radiation, which in this case has a FWHM of $235 \mathrm{meV}$. $(d)$ Convolution of the preceding curves. Note that the energy scale in $(d)$ is different from the other graphs.

$$
\Delta E(r, \varphi)=-\frac{h c}{2 d_{h k l} \sin \theta_{\mathrm{B}}} \varepsilon_{z z}^{\mathrm{h}}(r, \varphi)
$$

which is obtained by replacing the lattice spacing $d_{h k l}$ with $\left(1+\varepsilon_{z z}^{\mathrm{h}}\right) d_{h k l}$ in Bragg's law. We will show in $\$ 3$ that these assumptions lead to a good agreement with experiment.

The energy shift distribution of an analyser is obtained by dividing the surface of an analyser into a fine equally spaced Cartesian grid. Using (42), the values of $\Delta E$ are calculated for each of the grid points and their distribution is gained as a histogram of the points. The obtained curve is then convoluted with the computed Takagi-Taupin curve to gain the reflectivity curve of the whole analyser. In order to compare the calculated reflectivity curves with experiments, the curve has to be convoluted also with the bandwidth of the incoming radiation, Johann aberration and the finite source size effect when their contribution cannot be neglected.

It should be noted that, although used in this paper, the usage of Takagi-Taupin equations is not a necessity for the model presented here. The reflectivity curve of an analyser without angular compression can also be obtained with any other suitable method, e.g. the lamellar model (del Rio et al., 2004).

The composition of the final predicted reflectivity curve for a macroscopic spherically bent $\mathrm{Si}(660)$ crystal with a bending radius of $R=1 \mathrm{~m}$ and diameter $86 \mathrm{~mm}$ is presented in Fig. 3 .
The used Bragg angle was $88.7^{\circ}$, which corresponds to an X-ray energy of $9.7 \mathrm{keV}$. The energy shift distribution calculated with (42) is shown in Fig. 3(a). Fig. 3(b) shows the solution of the Takagi-Taupin equations with the strain field given by (22). In order to compare the reflectivity curve with the measurements in the following section, we have also taken into account the finite bandwidth of the incoming radiation in the measurement set-up, shown here for full width at half-maximum (FWHM) $235 \mathrm{meV}$ (Fig. 3c). The final reflectivity curve in Fig. 3(d) is obtained from the preceding components by a convolution. The Johann aberration and the error due to the finite source size can be neglected in our measurement set-up (both of the order of $10 \mathrm{meV}$ ).

\section{Comparison with measurements}

In order to verify the validity of the thin wafer model introduced in the previous chapter, we compare theoretical predictions against measured reflectivity curves of spherically bent analyser crystals with $R=1 \mathrm{~m}$ and $L=50 \mathrm{~mm}$. The measurements were made at the European Synchrotron Radiation Facility (ESRF) IXS beamline ID16. ${ }^{\mathbf{1}}$

The current mass-scale production of spherically bent crystals at ESRF relies on the anodic bonding technique (Wallis \& Pomerantz, 1969; Verbeni et al., 2005) with highly polished and thin $(<300 \mu \mathrm{m})$ wafers. In this paper we use $\mathrm{Si}(660)$ and $\mathrm{Si}(553)$ analysers as examples. With the current analyser manufacturing techniques, we have found $\mathrm{Si}(660)$ crystal qualities to be highly reproducible and chose three individual crystals as typical examples. The diffraction properties of the $\mathrm{Si}(553)$, on the other hand, have been found to vary more considerably. Possible reasons may include the lower crystallographic symmetry of the $\mathrm{Si}(553)$, which can cause the assumed rotation symmetry of the stress field to be an energetically unfavourable state.

The experiments were performed with analyser Bragg angles of $88.4-88.7^{\circ}$. The synchrotron beam was monochromated by a combination of $\mathrm{Si}(111)$ double-crystal and a $\mathrm{Si}(440)$ channel-cut monochromator to a bandwidth of $235 \mathrm{meV}$ at $9.69 \mathrm{keV}$, or $190 \mathrm{meV}$ at $8.77 \mathrm{keV}$ for $\mathrm{Si}(660)$ and $\mathrm{Si}(553)$ crystals, respectively. The beam was focused using a toroidal Rh-coated mirror to a spot size of $50 \mu \mathrm{m}$ in the dispersive (vertical) direction and $150 \mu \mathrm{m}$ in the non-disper-

\footnotetext{
${ }^{\mathbf{1}}$ ID16 was closed for operation in 2012 and the IXS spectroscopy research of electronic excitations continues at the new beamline ID20 as a part of the ESRF Upgrade Programme.
} 


\section{research papers}

sive (horizontal) direction to ensure negligible effects of a finite source size to the measured reflectivity curves.

\subsection{Reflectivity curves}

In X-ray spectroscopy, the reflectivity curves are also called resolution functions or (quasi)elastic lines. They were measured by scanning the incident photon energy across the range of energies corresponding to the analyser reflection, given by the Bragg angle and order of reflection, and using a Kapton foil as a scatterer.

For each analyser, we measured the reflectivity curves by using lead masks with circular apertures with diameters of 30 , 60 and $86 \mathrm{~mm}$. This way the dependence of the size of the active crystal surface could be studied. In addition to the analyser crystal reflectivity, the measured resolution functions include a broadening from the finite incident-beam bandwidth. To be able to compare experiment with theory, the theoretical reflectivity curves were convoluted with the incident bandwidth, which was approximated to be Gaussianshaped with a FWHM corresponding to the bandwidth quoted in the previous section.

A comparison of the measured and calculated resolution functions are presented in Fig. 4. The figure shows the resolution functions of three individual $\mathrm{Si}(660)$ analysers with three different active areas that are chosen by the mask aperture. Also shown are results for two $\mathrm{Si}(553)$ analysers with the same three mask apertures. A measurement of the reflectivity in absolute units was not attempted, and thus the reflectivities are given in arbitrary units, proportional to the reflected intensity recorded by the detector. The areas under the curves with different mask sizes are roughly proportional to the area of the unmasked part of the analyser, i.e. the collection solid angle (assuming the Kapton structure factor to be only weakly dependent on scattering angle within the measurement geometry).

The results for the mask aperture with diameter of $30 \mathrm{~mm}$ are close to the results from the Takagi-Taupin equation solver that assumes spherical bending without angular compression. Such a theoretical resolution function is characterized by an asymmetric lineshape with increased spectral weight towards the high-energy transfer side of the peak and a FWHM of roughly $0.4 \mathrm{eV}$ for $\mathrm{Si}(660)$ and $0.3 \mathrm{eV}$ for $\mathrm{Si}(553)$ when including the finite incident-beam bandwidth. However, the shape of the experimental resolution function shows increasing deviation from this shape with increasing analyser aperture. Relatively more spectral weight is collected on the low energy-transfer side when the analyser aperture is increased. This leads to an apparently symmetric lineshape with $60 \mathrm{~mm}$-diameter aperture [FWHM $0.5 \mathrm{eV}$ for $\mathrm{Si}(660)$, $0.4 \mathrm{eV}$ for $\mathrm{Si}(553)]$, and finally an asymmetric shape with a pronounced low-energy tail when the full analyser surface is used [FWHM $0.9 \mathrm{eV}$ for $\mathrm{Si}(553), 0.7 \mathrm{eV}$ for $\mathrm{Si}(553)]$. The same behaviour is manifested by experiments.

Our thin-wafer model can now predict the spectral shape as a function of analyser aperture as well. As can be seen, the model predicts correctly the shapes, relative intensities and
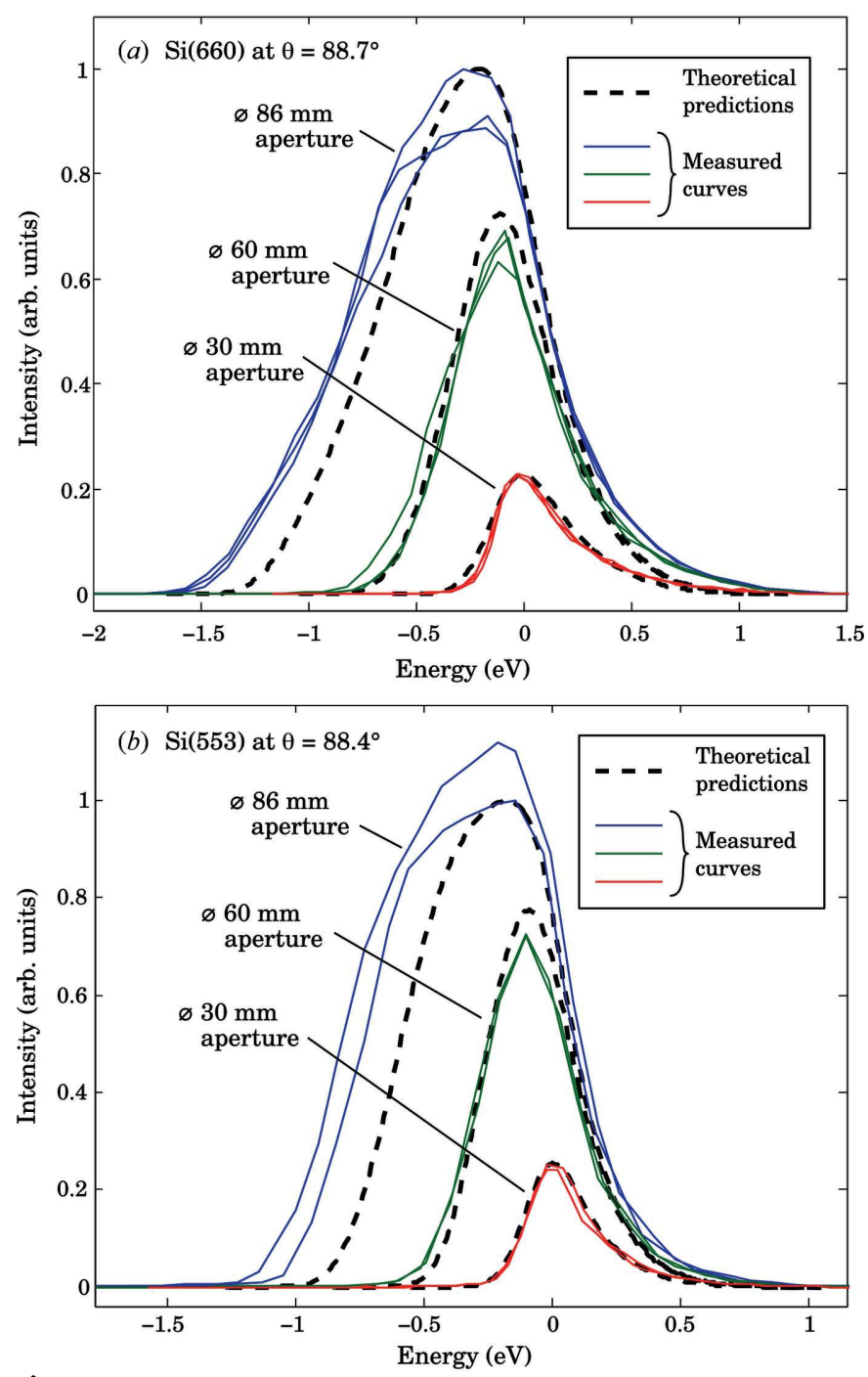

Figure 4

The predicted and measured reflectivity curves for $(a)$ three $\operatorname{Si}(660)$ and (b) two $\mathrm{Si}(553)$ analysers. Three different curves are shown for each analyser corresponding to different mask aperture sizes.

peak energy shifts for active surface areas of different sizes. This is true for both $\mathrm{Si}(660)$ and $\mathrm{Si}(553)$. A small discrepancy between experiment and calculation appears in the reflectivity curves at the low-energy side, especially when the full analyser surface is used, but the overall agreement is very good. This means that the presented simple model works well in predicting the diffraction properties of macroscopically large spherically bent wafers.

\section{Conclusions}

It has been known from measurements that the diffraction properties of macroscopically large elastically bent analyser crystals depend on the crystal size and bending radius. To improve the energy resolution in high-resolution X-ray spectroscopy, practical approaches have been made to increase the bending radius or decrease the analyser area (Verbeni et al., 2009; Collart et al., 2005). However, quantitative theoretical understanding of these properties as a function of the physical 
parameters has been lacking, and mainly rules-of-thumb have been used in their estimation. The well known Takagi-Taupin theory applied in the case of spherically bent crystals without angular compression usually predicts considerably narrower reflectivity curves, i.e. higher energy resolving power, than found in the experiment. This fundamental problem has slowed down the systematic development of spectrometers with the highest possible performance.

We have presented a treatment based on the theory of elasticity in the thin wafer approximation, to predict the diffraction properties of large-area analyser crystals. We apply the theory to spherically bent $\mathrm{Si}(660)$ and $\mathrm{Si}(553)$ analysers with diameters up to $100 \mathrm{~mm}$, bending radii of $1 \mathrm{~m}$, using Bragg angles of $88.4-88.7^{\circ}$. The proposed approach turns out to yield a precise yet simple model that can explain many of the observed properties of such elastically bent analysers. Most importantly, we can predict and explain quantitatively the dependence of the reflectivity curves as a function of active analyser crystal diameter between 30 and $86 \mathrm{~mm}$, which has been a fundamental unsolved problem up to now.

The fully realistic treatment would require finite-element calculations of the three-dimensional strain field of crystal wafers with finite thickness, combined with the Takagi-Taupin equation solver. However, the thin-wafer approximation used here is an important step towards a quantitative understanding of the properties of analyser crystals that are used in high-resolution X-ray spectroscopy at synchrotron and freeelectron laser facilities.

We are grateful to $\mathrm{C}$. Henriquet for expert advice and assistance. Beam time was provided by the European Synchrotron Radiation Facility. A-PH and SH were funded by the Academy of Finland (grants 1256211 and 1254065) and University of Helsinki research funds (grant 490076).

\section{References}

Alonso-Mori, R. et al. (2012). Proc. Natl Acad. Sci. 109, $19103-$ 19107.

Cauchois, Y. (1932). J. Phys. Rad. 3, 320-334.

Collart, E., Shukla, A., Gélébart, F., Morand, M., Malgrange, C., Bardou, N., Madouri, A. \& Pelouard, J.-L. (2005). J. Synchrotron Rad. 12, 473-478.

DuMond, J. W. M. \& Kirkpatrick, H. A. (1930). Rev. Sci. Instrum. 1, 88.

Erola, E., Eteläniemi, V., Suortti, P., Pattison, P. \& Thomlinson, W. (1990). J. Appl. Cryst. 23, 35-42.

Fast, L., Wills, J. M., Johansson, B. \& Eriksson, O. (1995). Phys. Rev. $B, \mathbf{5 1}, 17431-17438$.

Fister, T. T., Seidler, G. T., Wharton, L., Battle, A. R., Ellis, T. B., Cross, J. O., Macrander, A. T., Elam, W. T., Tyson, T. A. \& Qian, Q. (2006). Rev. Sci. Instrum. 77, 063901.

Gronkowski, J. (1991). Phys. Rep. 206, 1-41.

Groot, F. de \& Kotani, A. (2008). Core Level Spectroscopy of Solids. Boca Raton: CRC Press.

Hamos, L. von (1932). Naturwiss. 20, 705-706.

Hamos, L. von (1933). Ann. Phys. (Leipzig), 17, 716-724.

Hazemann, J.-L., Proux, O., Nassif, V., Palancher, H., Lahera, E., Da Silva, C., Braillard, A., Testemale, D., Diot, M.-A., Alliot, I., Del Net, W., Manceau, A., Gélébart, F., Morand, M., Dermigny, Q. \& Shukla, A. (2009). J. Synchrotron Rad. 16, 283-292.
Huotari, S., Albergamo, F., Vankó, G., Verbeni, R. \& Monaco, G. (2006). Rev. Sci. Instrum. 77, 053102.

Huotari, S., Pylkkänen, T., Verbeni, R., Monaco, G. \& Hämäläinen, K. (2011). Nat. Mater. 10, 489-493.

Huotari, S., Vankó, Gy., Albergamo, F., Ponchut, C., Graafsma, H., Henriquet, C., Verbeni, R. \& Monaco, G. (2005). J. Synchrotron Rad. 12, 467-472.

Johann, H. H. (1931). Z. Phys. 69, 185-206.

Johansson, T. (1932). Naturwissenschaften, 20, 758-759.

Johansson, T. (1935). Z. Phys. 82, 507-528.

Journel, L., El Khoury, L., Marin, T., Guillemin, R., Carniato, S., Avila, A., Delaunay, R., Hague, C. F. \& Simon, M. (2009). Rev. Sci. Instrum. 80, 093105.

Kleymenov, E., van Bokhoven, J. A., David, C., Glatzel, P., Janousch, M., Alonso-Mori, R., Studer, M., Willimann, M., Bergamaschi, A., Henrich, B. \& Nactegaal, M. (2011). Rev. Sci. Instrum. 82, 065107.

Kuriyama, M. (1967). J. Phys. Soc. Jpn, 23, 1369-1379.

Landau, L. D. \& Lifshitz, E. M. (1986). Theory of Elasticity. Oxford: Butterworth-Heinemann.

Llorens, I., Lahera, E., Delnet, W., Proux, O., Braillard, A., Hazemann, J.-L., Prat, A., Testemale, D., Dermigny, Q., Gelebart, F., Morand, M., Shukla, A., Bardou, N., Ulrich, O., Arnaud, S., Berar, J.-F., Boudet, N., Caillot, B., Chaurand, P., Rose, J., Doelsch, E., Martin, P. \& Solari, P. L. (2012). Rev. Sci. Instrum. 83, 063104.

Masciovecchio, C., Bergmann, U., Krisch, M., Ruocco, G., Sette, F. \& Verbeni, R. (1996). Nucl. Instrum. Methods Phys. Res. B, 117, 339 340 .

Mattern, B. A., Seidler, G. T., Haave, M., Pacold, J. I., Gordon, R. A., Planillo, J., Quintana, J. \& Rusthoven, B. (2012). Rev. Sci. Instrum. 83, 023901.

Nowacki, W. (1963). Dynamics of Elastic Systems. London: Chapman and Hall.

Pacold, J. I., Bradley, J. A., Mattern, B. A., Lipp, M. J., Seidler, G. T., Chow, P., Xiao, Y., Rod, E., Rusthoven, B. \& Quintana, J. (2012). J. Synchrotron Rad. 19, 245-251.

Penning, P. \& Polder, D. (1961). Philips Res. Rep. 16, 419-440.

Qian, J. Q. (2013). Private communication (http://xrstech.com).

Said, A. H., Sinn, H. \& Divan, R. (2011). J. Synchrotron Rad. 18, 492496.

Sakurai, Y., Itou, M., Barbiellini, B., Mijnarends, P. E., Markiewicz, R. S., Kaprzyk, S., Gillet, J.-M., Wakimoto, S., Fujita, M., Basak, S., Wang, Y.-J., Al-Sawai, W., Lin, H., Bansil, A. \& Yamada, K. (2011). Science, 332, 698-702.

Sanchez del Rio, M., Alianelli, L., Faenov, A. Y. \& Pikuz, T. (2004). Phys. Scr. 69, 297-302.

Sanchez del Rio, M., Ferrero, C. \& Mocella, V. (1997). Proc. SPIE, 3151, 312-323.

Schülke, W. (2007). Electron Dynamics Studied by Inelastic X-ray Scattering. Oxford University Press.

Shvyd'ko, Y. (2004). X-ray Optics: High-Energy-Resolution Applications. Berlin/Heidelberg: Springer-Verlag.

Shvyd'ko, Y., Hill, J. P., Burns, C. A., Coburn, D. S., Brajuskovic, B., Casa, D., Goetze, K., Gog, T., Khachatryan, R., Kim, J.-H., Kodituwakku, C. N., Ramanathan, M., Roberts, T., Said, A., Sinn, H., Shu, D., Stoupin, S., Upton, M., Wieczorek, M. \& Yavas, H. (2012). J. Electron Spectrosc. Relat. Phenom. 188, 140-149.

Takagi, S. (1962). Acta Cryst. 15, 1311-1312.

Takagi, S. (1969). J. Phys. Soc. Jpn, 26, 1239-1253.

Taupin, D. (1964). Bull. Soc. Fr. Miner. Crist. 87, 469-511.

Verbeni, R., Kocsis, M., Huotari, S., Krisch, M., Monaco, G., Sette, F. \& Vankó, G. (2005). J. Phys. Chem. Solids, 66, 2299-2305.

Verbeni, R., Pylkkänen, T., Huotari, S., Simonelli, L., Vankó, G., Martel, K., Henriquet, C. \& Monaco, G. (2009). J. Synchrotron Rad. 16, 469-476.

Wallis, G. \& Pomerantz, D. I. (1969). J. Appl. Phys. 40, 3946.

Welter, E., Machek, P., Dräger, G., Brüggmann, U. \& Fröba, M. (2005). J. Synchrotron Rad. 12, 448-454. 